\title{
Phase-Change Memory Electro-Thermal Analysis and Engineering Thanks to Enhanced Thermal Confinement
}

\author{
Anna Lisa Serra ${ }^{\mathrm{a}}$, Gauthier Lefevre ${ }^{\mathrm{b}}$, Olga Cueto ${ }^{\mathrm{a}}$, Guillaume Bourgeois ${ }^{\mathrm{a}}$, \\ Marie Claire Cyrille ${ }^{\mathrm{a}}$, Gabriele Navarro ${ }^{\mathrm{a}, *}$, Etienne Nowak ${ }^{\mathrm{a}}$, \\ ${ }^{a}$ Univ. Grenoble Alpes, F-38000 Grenoble, France CEA, LETI, MINATEC Campus, F-38054 Grenoble, France \\ ${ }^{b}$ CNRS-LTM Laboratoire des Technologies de la Micro electronique, 38054 Grenoble, France
}

\begin{abstract}
In this paper we compare the performances of $\mathrm{SiN}$ with respect to an optimized $\mathrm{SiC}$ encapsulation in Wall based Phase-Change Memory (PCM) integrating a Ge-rich Ge-Sb-Te alloy (GGST) suitable for high temperature stability in automotive applications. Thanks to the electrical characterization of $4 \mathrm{~kb}$ arrays, $3 \mathrm{D}$ electro-thermal simulations and TEM analyses performed on programmed devices, we demonstrate the higher programming efficiency in SiC-based PCM devices, thanks to the lower thermal conductivity of the optimized encapsulation. Indeed, the uniform temperature profile achieved in the active layer of $\mathrm{SiC}$ encapsulated PCM leads to a retention of one hour at $250{ }^{\circ} \mathrm{C}$. A theoretical model is here proposed to describe the electro-thermal behavior of the device, linking the electrical properties, such as the resistance as a function of current characteristics, to the thermal conductivity of the materials that constitute the device. Finally, thanks to our findings, we provide some guidelines to achieve drastic current reduction via the thermal engineering of the next generation PCM technology.
\end{abstract}

Keywords: Phase-Change Memory (PCM), thermal confinement, encapsulation layer, thermal conductivity, electro-thermal simulations.

\section{Introduction}

Phase-Change Memory (PCM) is the most mature among the emerging non-volatile memories (NVM) and it represents a reliable alternative to Flash technology. Indeed, it recently entered the market addressing Storage Class Memory (SCM) applications [1]. Moreover, PCM demonstrated to fulfill the automotive market requirements thanks to the phase-change material engineering. In particular, the use of Ge-rich Ge-Sb-Te alloys (GGST) ensures a data retention of ten years for temperatures higher than $150{ }^{\circ} \mathrm{C}$ [2]. Nevertheless, the main challenge to be overcome in PCM to target ultra low power applications in advanced technology nodes is the programming current reduction. Main efforts in recent years have been oriented to the development of scaled architectures [3, 4] and to the engineering of the active material [5, 6]. However,

^ This work was partially funded by European commission, French State and Auvergne-Rhone Alpes region through ECSEL project WAKEMEUP and French Nano2022 program.

${ }^{*}$ Corresponding author: G. Navarro (gabriele.navarro@cea.fr)

Email addresses: annalisa.serra@cea.fr (Anna Lisa Serra), gauthier.lefevre@cea.fr (Gauthier Lefevre), olga.cueto@cea.fr (Olga Cueto), guillaume.bourgeois@cea.fr (Guillaume Bourgeois), marie-claire.cyrille@cea.fr (Marie Claire Cyrille), gabriele.navarro@cea.fr (Gabriele Navarro), etienne.nowak@cea.fr (Etienne Nowak) 
the main part of the power used during the programming operations of a PCM device is represented by the heat losses [1], making the PCM performance to be far from the adiabatic limit represented by a perfect thermal isolation and zero losses [7]. Only in the last years, with the increasing in maturity of the PCM technology, the engineering of thermal barriers and of the thermal conductivity of the surrounding dielectrics became the object of preliminary studies confirming the huge improvement achievable already in analytic devices [8, 9] and demonstrating its even higher necessity in ultra scaled PCM [10]. In this framework, we propose the investigation of the thermal improvement in heater-based (i.e. "Wall") state-of-the-art PCM structures [4] based on GGST alloy. Thanks to the introduction of an optimized SiC encapsulation layer featuring low thermal conductivity we highlight the benefit of an improved thermal isolation, with respect to standard SiN, by statistical electrical results obtained on $4 \mathrm{~kb}$ arrays. In particular, we show the higher efficiency of the RESET operation (i.e. amorphization), which leads to a more uniform amorphous region and an improved data retention [11]. The electrical results are validated here by 3D electro-thermal simulations and Transmission Electron Microscopy (TEM) observations combined to Energy-dispersive X-ray (EDX) spectroscopy performed on programmed devices. Finally, we present a physical model to correlate the resistance-vs-current (RI) characteristic to the thermal conductivity of the dielectrics surrounding the cell, validating it thanks to the electrical and thermal data acquired. We provide a clear demonstration of the importance of the thermal properties engineering not only of the phase-change layer (PCL) but also of the dielectrics surrounding the heater, and we show the correlation of such properties to the final electrical characteristics of the PCM device.

\section{Device fabrication and electrical characterization}

The thermal conductivity of the $\mathrm{SiN}$ and $\mathrm{SiC}$ layers used as encapsulation were measured by $3 \omega$ method 12 ] giving a value of $0.41 \mathrm{Wm}^{-1} \mathrm{~K}^{-1}$ for $\mathrm{SiC}$ and $1.39 \mathrm{Wm}^{-1} \mathrm{~K}^{-1}$ for $\mathrm{SiN}$ [1]. The $\mathrm{SiN}$ and $\mathrm{SiC}$ encapsulation layers were deposited by Plasma Enhanced Chemical Vapor Deposition (PECVD) on the sides of the PCM device after the etching of the PCL (Fig. 1), based on a GGST alloy integrated by sputtering from single target. The PCM devices were fabricated in the Back-End-Of-Line (BEOL) process of our LETI Memory Advanced Demonstrator (MAD), based on $130 \mathrm{~nm}$ CMOS technology, used for statistical electrical parameters evaluation thanks to embedded $4 \mathrm{~kb}$ arrays. The used BEOL process thermal budget is estimated in the order of about 30 minutes at $400{ }^{\circ} \mathrm{C}$. For our electrical and physico-chemical analyses we considered devices with a wall width of about $80 \mathrm{~nm}$ and in the following we will refer to the devices by the material of their encapsulation (i.e. $\mathrm{SiC}$ or $\mathrm{SiN}$ ).

The RI characteristics were measured for both $\mathrm{SiC}$ and $\mathrm{SiN}$ devices in $4 \mathrm{~kb}$ arrays. After a seasoning procedure of ten SET/RESET cycles, the devices were initialized in the SET state (i.e. crystalline phase) with a pulse having a long fall time (SET-init) in order to achieve the lowest possible SET resistance state (i.e. rise/width/fall times equal to $10 \mathrm{~ns} / 300 \mathrm{~ns} / 10 \mu \mathrm{s}$ ) for both $\mathrm{SiC}$ and $\mathrm{SiN}$ cells. The gradual resistance increase towards the RESET state is obtained with a staircase-up sequence of squared pulses (SP) of increasing voltage (i.e. current) amplitude, having rise/width/fall time equal to $10 \mathrm{~ns} / 300 \mathrm{~ns} / 10 \mathrm{~ns}$, pre-programming the device in the SET state with a SET-init pulse before each SP. The results are reported in Fig. 2. The different starting SET resistance, obtained by the specific procedure described above, is likely related to a different Ge expulsion during the forming operation [13] and the likely presence of residual amorphous regions due to the different thermal isolation, as it will be confirmed in following analyses. The different starting resistance, influences the "melting current" ( $I_{m}$ represented by the first detection of resistance increase in the RI curve), as already reported in previous works 14]. In particular, SiN based devices feature a melting current about $100 \mu \mathrm{A}$ lower than the $\mathrm{SiC}$ based one. Both RI curves present two different slopes after reaching $\mathrm{I}_{\mathrm{m}}$, identified as $s_{1}$ and $s_{2}$ and reported in Table 1, highlighting a steeper resistance increase in $\mathrm{SiC}$ (i.e. higher $s_{1}$ and $s_{2}$ ).

An isochronal annealing of one hour at increasing temperatures (from $150{ }^{\circ} \mathrm{C}$ up to $250{ }^{\circ} \mathrm{C}$ ) was applied to test the data retention performances comparing $\mathrm{SiC}$ and $\mathrm{SiN}$. The results are reported in Fig. 3 and are performed on a population of $2 \mathrm{~kb}$ (half $4 \mathrm{~kb}$ matrix) for both SET and RESET states. The SET state in both devices faces a known SET drift [15] up to $150{ }^{\circ} \mathrm{C}$ before to trigger the crystallization mechanism at higher temperature with a consequent resistance decrease. $\mathrm{SiC}$ devices show a very good $\mathrm{SET}$ and RESET retention 


\begin{tabular}{c|c|c} 
material & $\begin{array}{c}s_{1} \\
{[\mathrm{dec} / \mathrm{mA}]}\end{array}$ & $\begin{array}{c}s_{2} \\
{[\mathrm{dec} / \mathrm{mA}]}\end{array}$ \\
\hline $\mathrm{SiC}$ & $3.41 \pm 0.36$ & $1.74 \pm 0.01$ \\
$\mathrm{SiN}$ & $1.96 \pm 0.03$ & $0.95 \pm 0.01$
\end{tabular}

Table 1. Slopes $\left(s_{1}, s_{2}\right)$ and variance for both $\mathrm{SiC}$ and $\mathrm{SiN}$ devices, obtained from the RI characteristics reported in Fig. 2

up to $250^{\circ} \mathrm{C}$, while SiN devices programmed in RESET state face a $25 \%$ of failures after such annealing. As demonstrated in the following, the different behavior is likely related to a different in the amorphous region morphology between $\mathrm{SiN}$ and $\mathrm{SiC}$ which is achieved in the device active volume (i.e. programmed volume) after programming it in the RESET state.

\section{Electro-thermal simulations and TEM/EDX analyses}

We performed 3D electro-thermal simulations in COMSOL Multiphysics in order to analyze the impact of the encapsulation on the PCM cell, introducing SiC and SiN thermal conductivities previously measured. Methods and parameters used are described in previous works [16]. The different initialization is taken into account by tuning the electrical conductivity of the PCL to get a match between the simulated and the experimental SET resistance. Being the structure symmetric with respect to the z-axis in the zy-plane (see Fig. 1), only half of the structure is simulated. The temperature profile achieved in the two systems is reported in Fig. 4. The volume and the shape of the region reaching a temperature higher than $930 \mathrm{~K}$ (i.e. melting temperature of the phase-change material) is not the same in the two structures. In $\mathrm{SiC}$ the melted region in the PCL is larger and more uniform along the y axis, while in SiN the temperature decreases moving from the center $(\mathrm{y}=0$ a.u. $)$ to the heater/encapsulation interface $(\mathrm{y}=0.4$ a.u.). This explains why $\mathrm{SiC}$ has a higher resistance when programmed at $1.2 \mathrm{~mA}$ in RESET state in Fig. 2, being the amorphized volume thickness more uniform in $\mathrm{SiC}$ than in SiN. A more specific analysis is presented in the next subsections for both $\mathrm{SiN}$ and $\mathrm{SiC}$, describing in detail how the temperature profile changes in the PCM device increasing the programming current. In this investigation, the value of the simulated $\mathrm{I}_{\mathrm{m}}$ is represented by the current at which the PCL starts to melt.

\subsection{SiN encapsulation}

In order to understand the temperature profile achieved in the device, we analyzed the isotherms reaching $930 \mathrm{~K}$ in both the PCL and the heater and measured their distance from the PCL/heater interface $\left(\mathrm{h}_{930 \mathrm{~K}}\right)$. The evolution of $\mathrm{h}_{930 \mathrm{~K}}$ in the PCL and in the heater at the center of the cell (i.e. $\mathrm{y}=0$ a.u.) and at the PCL/encapsulation interface (i.e. $\mathrm{y}=0.4$ a.u.) are reported as a function of the applied current in a.u. since the simulated device has a different width than the measured one (Fig. [5a). SiN based PCM starts to melt at the center of the heater width for an applied current of 0.4 a.u., whereas the melting at the PCL/encapsulation interface is achieved at 0.6 a.u.. Only at high current the melted thickness becomes homogeneous on the heater surface, and the heat propagation in the heater becomes really important. The position of the maximum temperature $\left(\mathrm{T}_{\max }\right)$ along the $\mathrm{z}$-axis during the programming operations in both previous analyzed regions of the cell (i.e. $\mathrm{y}=0$ a.u. and $\mathrm{y}=0.4 \mathrm{a} . \mathrm{u}$ ) is reported in Fig. [5b. At low current, $\mathrm{T}_{\max }$ is localized inside the PCL at both y positions, while at increasing current the scenario changes: in the center we observe a gradual localization of $\mathrm{T}_{\max }$ at the $\mathrm{PCL} /$ heater interface, while at the $\mathrm{PCL} /$ encapsulation interface $\mathrm{T}_{\max }$ is already inside the heater. TEM/EDX images acquired along zx and zy plane of SiN devices programmed in the RESET state are reported in Fig. 6. The EDX maps along the zy plane (Fig. 6a) shows a not uniform active region on the top of the heater, well evident in the Sb map, compatible with what observed in simulations. On the contrary, along the zx plane (Fig. 6b), the elemental distribution results to be more homogeneous due to the signal integration along the whole lamella thickness, which confirms a higher elemental profile change mainly only along the y-axis. A slight evidence of the shape of the active region is however present in the $\mathrm{Sb}$ and Te maps. 


\subsection{SiC encapsulation}

Same analyses performed for $\mathrm{SiN}$ are reported here for $\mathrm{SiC}$ in Fig. $\mathbf{7}$ (simulations) and Fig. $\mathbf{8}$ (TEM/EDX). The melting of the PCL is reached for both center and border at 0.5 a.u.. with a $\mathrm{h}_{930 \mathrm{~K}}$ evolution at increasing current almost identical at both y positions. This confirms that the thickness of the melted region (i.e. that becomes amorphous volume after the RESET pulse application) is uniform over the heater/PCL interface along the y-direction, as expected from Fig. 4b. The position of $\mathrm{T}_{\max }$ (Fig. $7 \mathrm{~b}$ ) at increasing current is also confirming a good homogeneity of the temperature distribution along the y-axis in the cell (i.e. well matched behavior between center and border). As observed for SiN, at low current $\mathrm{T}_{\max }$ is localized in the PCL, while increasing the current $T_{\max }$ shifts inside the heater. The TEM/EDX of a RESET cell along zy plane (Fig. [8 a) shows a uniform elemental distribution over the heater/PCL interface in all the Ge, Sb and Te maps. Moreover, the active volume is clearly evidenced along zy plane (Fig. $8 \mathrm{~b}$ ) since uniformly achieved on the whole heater/PCL surface (y-direction) and along the whole lamella thickness (of about one hundred $\mathrm{nm}$ ), with an elemental distribution compatible with previous works [17, 13]. Such homogeneity, achieved thanks to $\mathrm{SiC}$ encapsulation, is in agreement with simulations results.

\section{Electro-thermal theoretical analysis and discussions}

The double slope behavior evidenced in the RI characteristic of both devices in Fig. 2, can be correlated to the different programming scenario that is achieved in the cell for different current amplitudes. As observed in simulations, the transition of the temperature profile from the PCL to the heater, is the origin of the double slope. In first approximation we can describe the electro-thermal behavior of the cell in $2 \mathrm{D}$ along the zy plane, as previously reported [4]. We can consider that the resistance value $R$ achieved in the PCM device after the pulse application can be expressed as a function of the amorphous thickness obtained in the cell:

$$
R \simeq \int_{0}^{h_{a m}} \rho_{a m} \frac{d z}{\pi z w} \propto \ln \left(h_{a m}\right)
$$

where $h_{a m}$ is the amorphous thickness, $\rho_{a m}$ the resistivity of the amorphous phase and $w$ the width of the heater along the y direction. We considered a uniform semi-cylindrical amorphous shape over the heater. In such system, the heat distribution is driven by the stationary heat transfer equation, that can be simplified as:

$$
\frac{\partial^{2} T}{\partial z^{2}} \simeq-\frac{\rho_{O N} I^{2}}{k_{t h, e f f}}
$$

where $\rho_{O N}$ represents the device resistivity when the PCL is melted during the pulse application, $T$ is the temperature along the z-axis, $k_{t h, e f f}$ is the effective thermal conductivity associated to the overall system, while $I$ is the current applied to the device and which has the role to induce a specific temperature profile inside the cell thanks to the achieved current density $J$ at the PCL/heater interface $(I=J w t$ with $t$ the heater thickness along the $\mathrm{x}$-axis). Considering the height of the melted region equal to the height of the amorphous final region $\left(\mathrm{h}_{930 \mathrm{~K}}=h_{a m}\right)$ we can write from equation eq. (2):

$$
\Delta T_{i} \propto \frac{I^{2}}{k_{t h, e f f}} h_{a m}^{2}
$$

where $\Delta T_{i}$ represents the temperature increase achieved at the heater/PCL interface (with respect to room temperature), and giving rise to the following correlation between $h_{a m}$ and $I$ :

$$
\frac{d h_{a m}}{d I} \propto \frac{\sqrt{\Delta T_{i} k_{t h, e f f}}}{I^{2}}
$$

Introducing the known correlation between power $(P)$ and temperature we have:

$$
\Delta T_{i}=R_{T H} P \propto R_{T H} I^{2}
$$


where $R_{T H}$ is the global thermal resistance of the device. We can finally simplify eq. (4), considering the linear proportionality between $R_{T H}$ and the reciprocal of the effective thermal conductivity of the system $k_{t h, e f f}$ :

$$
\frac{d h_{a m}}{d I} \propto \frac{R_{T H} \sqrt{k_{t h, e f f}}}{\sqrt{\Delta T_{i}}} \propto \frac{1}{\sqrt{k_{t h, e f f}}}
$$

This correlation is in agreement with expectations, namely that the thickness of the amorphous region achieved in the cell when a specific current is applied increases when the thermal conductivity of the surrounding materials is reduced. Therefore, since the resistance $R$ depends on $h_{a m}$, we can finally correlate the slope of the RI curve of Fig. 2 (i.e. $s_{1}$ or $s_{2}$ ) to the thermal conductivity of the system:

$$
s=\frac{d \ln (R)}{d I}=\frac{d \ln (R)}{d h_{a m}} \frac{d h_{a m}}{d I} \propto \frac{1}{\sqrt{k_{t h, e f f}}}
$$

The electrical results obtained from $4 \mathrm{~kb}$ were performed on totally equivalent devices, except for the different encapsulation layer. This allows us to validate the relation in eq. (7), by calculating the ratios between the slopes in Table 1 and correlating them with the ratios between the measured thermal conductivities. Therefore, the ratio between the two $s_{1}$ is $\mathrm{SiC}$ and $\mathrm{SiN}$, and the two $s_{2}$ should depend only on the ratio between the thermal conductivities of the two materials and this is confirmed by the following calculation:

$$
\frac{s_{1, S i C}}{s_{1, S i N}}=\frac{s_{2, S i C}}{s_{2, S i N}}=\frac{\sqrt{k_{t h, S i N}}}{\sqrt{k_{t h, S i C}}} \sim 1.8
$$

This evidences that the difference between the evolution of SiC and SiN RI curves is strongly related to the value of the thermal conductivity of the encapsulation layer which is in direct contact with the PCL and the heater. Therefore, the choice of the encapsulation layer is fundamental in the tuning of the zy plane temperature profile of a Wall based PCM and further optimization can allow an even steeper slope in the RI curve, leading to a current reduction for the RESET operation.

The lower uniformity along y-axis observed in SiN devices can explain the lower $\mathrm{I}_{\mathrm{m}}$ found in Fig. 2 2 with respect to $\mathrm{SiC}$. Indeed, some amorphous residuals featuring low thermal conductivity could be generated by such non-homogeneous temperature profile, reducing the current needed to melt the active volume in the PCL. The amorphous residuals are compatible also with the increased SET resistance in SiN devices with respect to $\mathrm{SiC}$.

An other validation of eq. (77) can be obtained by comparing the transition from $s_{1}$ to $s_{2}$ observed in both encapsulation layers. Our hypothesis, confirmed in simulations, supports a transition of $\mathrm{T}_{\max }$ from the PCL at low currents towards the heater at higher currents. This is an effect which depends on both the phase-change material electrical resistivity evolution in temperature and the amount of PCL that triggers the melting during the programming operations. Based on such hypothesis, we can consider that the main change between the two regimes, in terms of materials surrounding $T_{\max }$, would be along the $\mathrm{x}$-axis, since along $\mathrm{y}$-axis the encapsulation dielectric remains unchanged. Indeed, when $\mathrm{T}_{\max }$ is inside the PCL, it is surrounded by the phase-change material itself along $\mathrm{x}$-axis as it is visible in the $\mathrm{zx}$ scheme of Fig. 1] In such case the thermal conductivity of the PCL influences $s_{1}$. On the contrary, when $\mathrm{T}_{\max }$ is localized inside the heater, it is surrounded by a SiN based dielectric (referred as "DIEL." in the scheme of Fig. 1) along the X-axis, with an effect on $s_{2}$. Such $\mathrm{SiN}$ is of the same nature as the one used for the encapsulation (i.e. PECVD) with equivalent thermal conductivity. The ratio between $s_{1}$ and $s_{2}$ is equivalent in both $\mathrm{SiC}$ and $\mathrm{SiN}$ devices:

$$
\frac{s_{1, S i C}}{s_{2, S i C}} \sim \frac{s_{1, S i N}}{s_{2, S i N}} \sim 2
$$

This confirms the hypothesis of the equivalent origin of the transition between the two slopes in both encapsulation layers, namely the change of position of $\mathrm{T}_{\max }$ at increasing current. Following our reasoning it would mean that:

$$
\frac{s_{1}}{s_{2}}=\frac{\sqrt{k_{t h, S i N}}}{\sqrt{k_{t h, P C L}}} \sim 2 \quad \rightarrow \quad k_{t h, P C L} \sim 0.37 \mathrm{~W} / \mathrm{mK}
$$


This result is in agreement with previous works reporting the thermal conductivity of fcc GeSbTe alloys [18, 19]. Considering the heterogeneity of GGST layer after BEOL process, facing phases segregation (i.e. formation of fcc GeSbTe phase and Ge phase) as reported in [13, 17], the thermal conductivity value found in our calculation is in agreement with the presence of grain boundaries and residual amorphous regions in the PCL, outside the active volume. Moreover, being the thermal conductivity of crystalline Ge higher than fcc GeSbTe, the thermal transport in the GGST system should be driven by the GeSbTe phase segregated.

This last result highlights the high importance of the engineering of the dielectrics surrounding the heater element in a Wall structure, in order to make the programming operation more efficient. For a given PCL, the encapsulation layer thermal conductivity influences the steepness of $s_{1}$ while the thermal conductivity of the dielectrics surrounding the heater influences $s_{2}$. Therefore, in a Wall PCM structure featuring a highly resistive heater element, with a $\mathrm{T}_{\max }$ localized inside the heater at each programming current, the slope $s_{2}$ dominates the RI characteristic and the engineering of the dielectrics surrounding the heater becomes fundamental for drastic current reduction.

\section{Conclusion}

In this work we report the improved electrical performances in Wall based state-of-the-art 4kb PCM arrays thanks to the engineering of the device encapsulation introducing an optimized $\mathrm{SiC}$ layer featuring low thermal conductivity. Thanks to a more homogeneous amorphous volume obtained in the PCL, we demonstrate an improved data retention in GGST alloys of more than one hour at $250{ }^{\circ} \mathrm{C}$. Moreover, the programming operation becomes more efficient being the SET to RESET transition steeper due to the improved thermal confinement. The results are supported by 3D electro-thermal simulations and TEM/EDX analyses. Finally, a theoretical model is proposed revealing the correlation between the slopes of the RI characteristics and the thermal conductivities of the materials constituting the device. The model is validated by using the measured thermal conductivities of the dielectrics surrounding the PCL and the heater, and the PCL thermal conductivity available in literature. Finally, we provide a demonstration of the strong impact of the dielectrics thermal conductivities and of the thermal boundaries surrounding the heater element, providing a path towards ultra low power next generation PCM devices.

\section{References}

[1] S. W. Fong, C. M. Neumann, H.-S. P. Wong, Phase-change memory-towards a storage-class memory, IEEE Transactions on Electron Devices 64 (11) (2017) 4374-4385.

[2] P. Cappelletti, R. Annunziata, F. Arnaud, F. Disegni, A. Maurelli, P. Zuliani, Phase Change Memory for automotive grade embedded NVM applications, Journal of Physics D: Applied Physics 53 (19) (2020) 193002.

[3] I. S. Kim, S. L. Cho, D. H. Im, E. H. Cho, D. H. Kim, G. H. Oh, D. H. Ahn, S. O. Park, S. W. Nam, J. T. Moon, C. H. Chung, High performance PRAM cell scalable to sub-20nm technology with below 4F2 cell size, extendable to dram applications, in: 2010 Symposium on VLSI Technology, 2010, pp. 203-204.

[4] M. Boniardi, A. Redaelli, C. Cupeta, F. Pellizzer, L. Crespi, G. D'Arrigo, A. L. Lacaita, G. Servalli, Optimization metrics for Phase Change Memory (PCM) cell architectures, in: 2014 IEEE International Electron Devices Meeting, IEEE, 2014, pp. 29-1.

[5] J. H. Park, S.-W. Kim, J. H. Kim, Z. Wu, S. L. Cho, D. Ahn, D. H. Ahn, J. M. Lee, S. U. Nam, D.-H. Ko, Reduction of reset current in phase change memory devices by carbon doping in GeSbTe films, Journal of Applied Physics 117 (11) (2015) 115703.

[6] H. L. Lung, Y. H. Ho, Y. Zhu, W. C. Chien, S. Kim, W. Kim, H. Y. Cheng, A. Ray, M. Brightsky, R. Bruce, C. W. Yeh, C. Lam, A novel low power phase change memory using inter-granular switching, in: 2016 IEEE Symposium on VLSI Technology, 2016, pp. 1-2.

[7] F. Xiong, E. Yalon, A. Behnam, C. M. Neumann, K. L. Grosse, S. Deshmukh, E. Pop, Towards ultimate scaling limits of Phase Change Memory, in: 2016 IEEE International Electron Devices Meeting (IEDM), 2016, pp. 4.1.1-4.1.4. doi:10.1109/IEDM.2016.7838342

[8] K. Ren, Y. Cheng, X. Chen, K. Ding, S. Lv, W. Yin, X. Guo, Z. Ji, Z. Song, Carbon layer application in Phase Change Memory to reduce power consumption and atomic migration, Materials Letters 206 (2017) 52-55.

[9] S. W. Fong, C. M. Neumann, E. Yalon, M. M. Rojo, E. Pop, H.-S. P. Wong, Dual-layer dielectric stack for thermally isolated low-energy Phase Change Memory, IEEE Transactions on Electron Devices 64 (11) (2017) 4496-4502.

[10] S. Muneer, A. Gokirmak, H. Silva, Vacuum-insulated self-aligned nanowire Phase Change Memory devices, IEEE Transactions on Electron Devices 62 (5) (2015) 1668-1671. 
[11] A. Serra, O. Cueto, N. Castellani, J. Sandrini, G. Bourgeois, N. Bernier, M. Cyrille, J. Garrione, M. Bernard, V. Beugin, et al., Outstanding Improvement in $4 \mathrm{~Kb}$ Phase-Change Memory of Programming and Retention Performances by Enhanced Thermal Confinement, in: 2019 IEEE 11th International Memory Workshop (IMW), IEEE, 2019, pp. 1-4.

[12] D. G. Cahill, R. O. Pohl, Thermal conductivity of amorphous solids above the plateau, Physical review B 35 (8) (1987) 4067.

[13] V. Sousa, G. Navarro, N. Castellani, M. Coue, O. Cueto, C. Sabbione, P. Noe, L. Perniola, S. Blonkowski, P. Zuliani, R. Annunziata, Operation fundamentals in 12Mb Phase Change Memory based on innovative Ge-rich GST materials featuring high reliability performance, in: 2015 Symposium on VLSI Technology (VLSI Technology), 2015, pp. T98-T99.

[14] A. L. Lacaita, A. Redaelli, The race of Phase Change Memories to nanoscale storage and applications, Microelectronic engineering 109 (2013) 351-356.

[15] N. Ciocchini, E. Palumbo, M. Borghi, P. Zuliani, R. Annunziata, D. Ielmini, Modeling resistance instabilities of set and reset states in phase change memory with Ge-rich $\mathrm{Ge}_{2} \mathrm{Sb}_{2} \mathrm{Te}_{5}$, IEEE Transactions on Electron Devices 61 (6) (2014) $2136-2144$.

[16] O. Cueto, V. Sousa, G. Navarro, S. Blonkowski, Coupling the phase-field method with an electrothermal solver to simulate phase change mechanisms in PCRAM cells, in: 2015 International Conference on Simulation of Semiconductor Processes and Devices (SISPAD), IEEE, 2015, pp. 301-304.

[17] P. Zuliani, E. Varesi, E. Palumbo, M. Borghi, I. Tortorelli, D. Erbetta, G. D. Libera, N. Pessina, A. Gandolfo, C. Prelini, L. Ravazzi, R. Annunziata, Overcoming temperature limitations in Phase Change Memories with optimized $\mathrm{Ge}_{\mathrm{x}} \mathrm{Sb}_{\mathrm{y}} \mathrm{Te}_{\mathrm{z}}$, IEEE Transactions on Electron Devices 60 (12) (2013) 4020-4026.

[18] H.-K. Lyeo, D. G. Cahill, B.-S. Lee, J. R. Abelson, M.-H. Kwon, K.-B. Kim, S. G. Bishop, B.-k. Cheong, Thermal conductivity of phase-change material $\mathrm{Ge}_{2} \mathrm{Sb}_{2} \mathrm{Te}_{5}$, Applied Physics Letters 89 (15) (2006) 151904.

[19] K. S. Siegert, F. R. L. Lange, E. R. Sittner, H. Volker, C. Schlockermann, T. Siegrist, M. Wuttig, Impact of vacancy ordering on thermal transport in crystalline phase-change materials, Reports on Progress in Physics 78 (1) (2014) 013001.
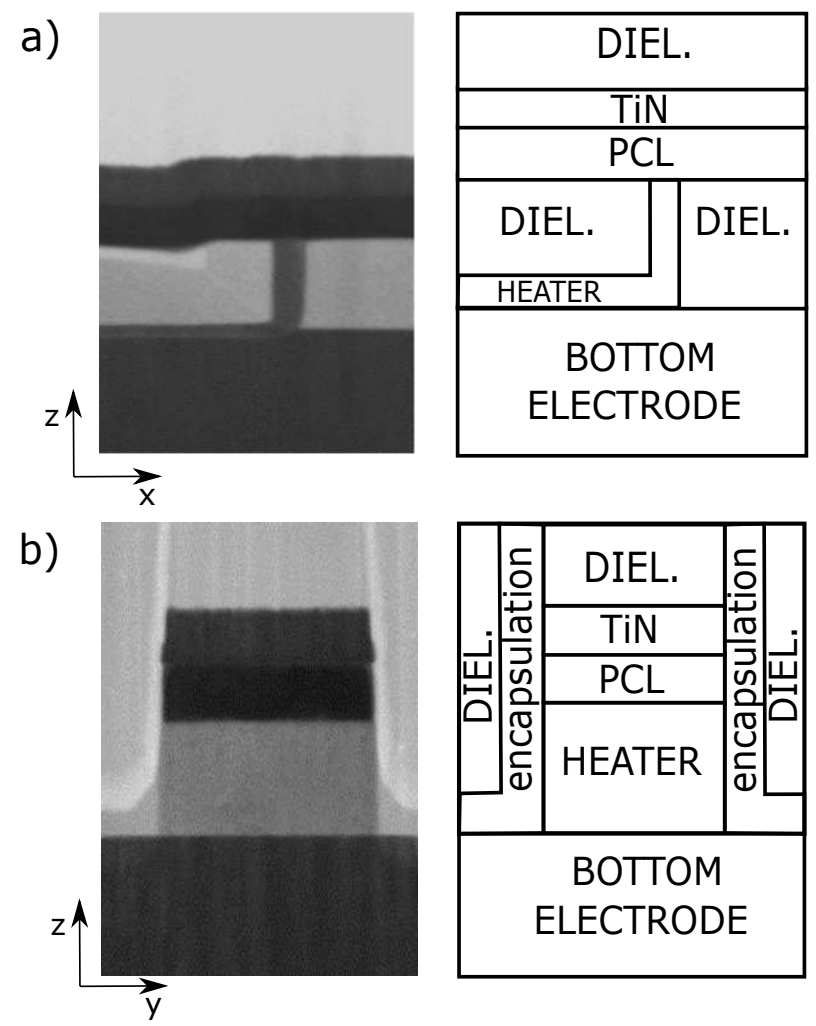

Fig. 1. Simplified description of our heater based PCM device. TEM images performed on the zx (a) and zy (b) planes on a PCM device with a nominal heater width of $300 \mathrm{~nm}$ are reported along with the structure description. The encapsulation layer ( $\mathrm{SiN}$ or $\mathrm{SiC}$ ), the heater, the phase-change layer (PCL) and the and dielectrics (DIEL.) are evidenced. 


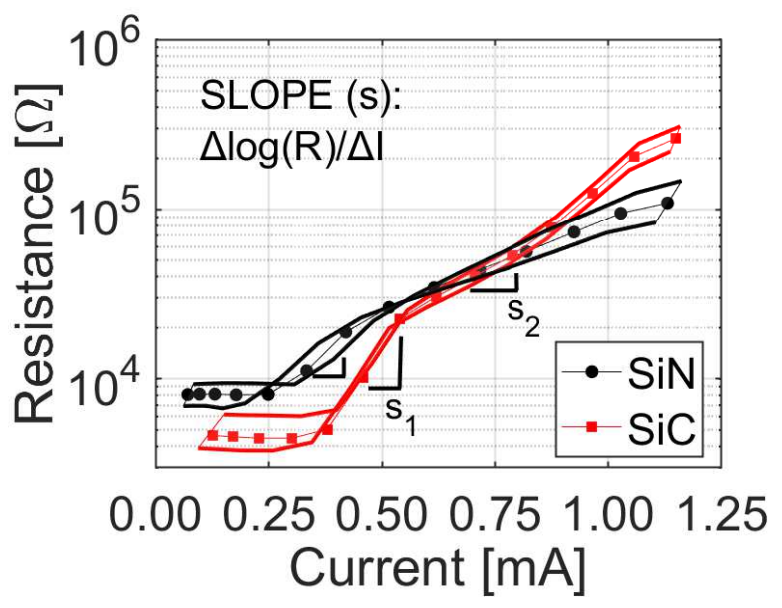

Fig. 2. Resistance as a function of the programming current (RI) for $4 \mathrm{~kb}$ PCM arrays based on GGST alloy with respectively $\mathrm{SiN}$ and $\mathrm{SiC}$ as encapsulation layers. The slopes $s_{1}$ and $s_{2}$ are calculated as $\ln (R) / d I$ for both devices and reported in Table 1
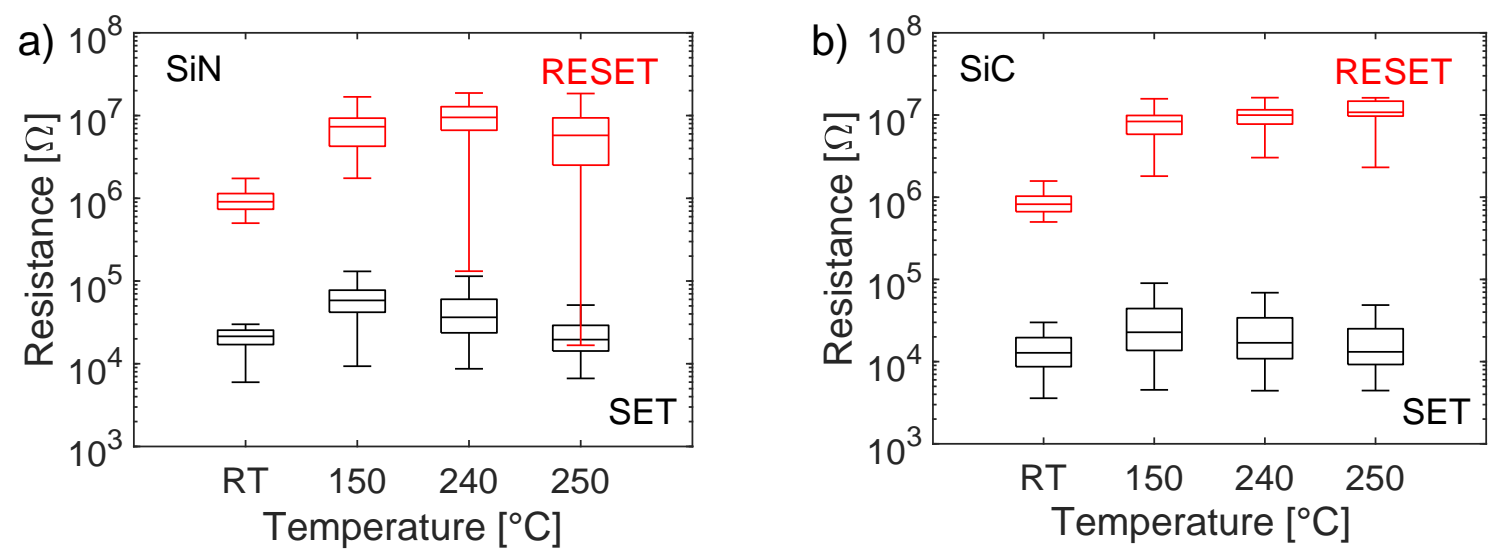

Fig. 3. SET and RESET data retention performed by isochronal annealing of one hour at increasing temperatures for both $\mathrm{SiN} \mathrm{(a)} \mathrm{and} \mathrm{SiC} \mathrm{(b)} \mathrm{where} \mathrm{the} \mathrm{minimum} \mathrm{resistance} \mathrm{value} \mathrm{in} \mathrm{the} \mathrm{box} \mathrm{represents} \mathrm{the} \mathrm{first} \mathrm{quantile,} \mathrm{while} \mathrm{the} \mathrm{bar} \mathrm{covers} \mathrm{the} \mathrm{whole}$ range of data measured. About the $25 \%$ of SiN based devices face a RESET failure at $250{ }^{\circ} \mathrm{C}$ while $\mathrm{SiC}$ ones retain RESET up to more than one hour at the same temperature. The SET resistance faces a resistance drift for both $\mathrm{SiC}$ and $\mathrm{SiN}$ up to $150{ }^{\circ} \mathrm{C}$, compatibly with previous works 15 , followed by a crystallization. 
a)

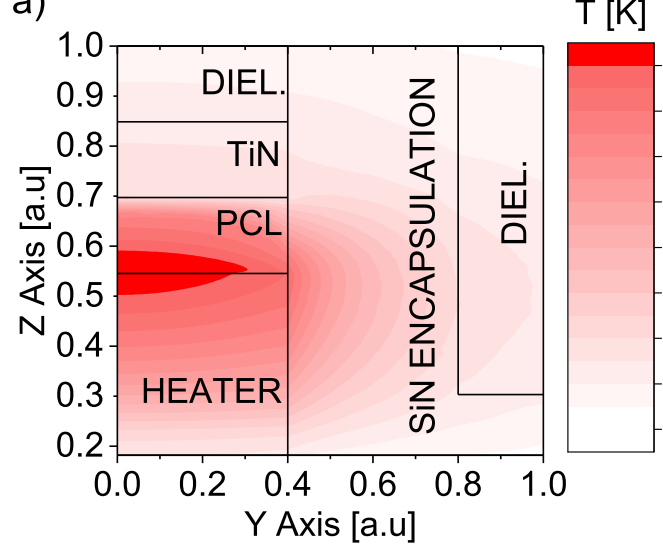

b)

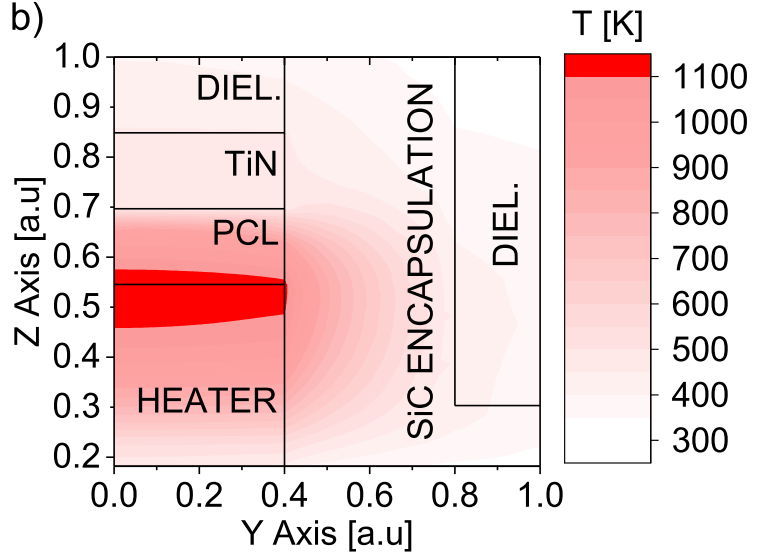

Fig. 4. Temperature profile simulated for both $\mathrm{SiN}(\mathrm{a})$ and $\mathrm{SiC}$ (b) for same applied current. The SiC device presents a more uniform temperature profile along the y-direction and a temperature gradient oriented along the z-axis.
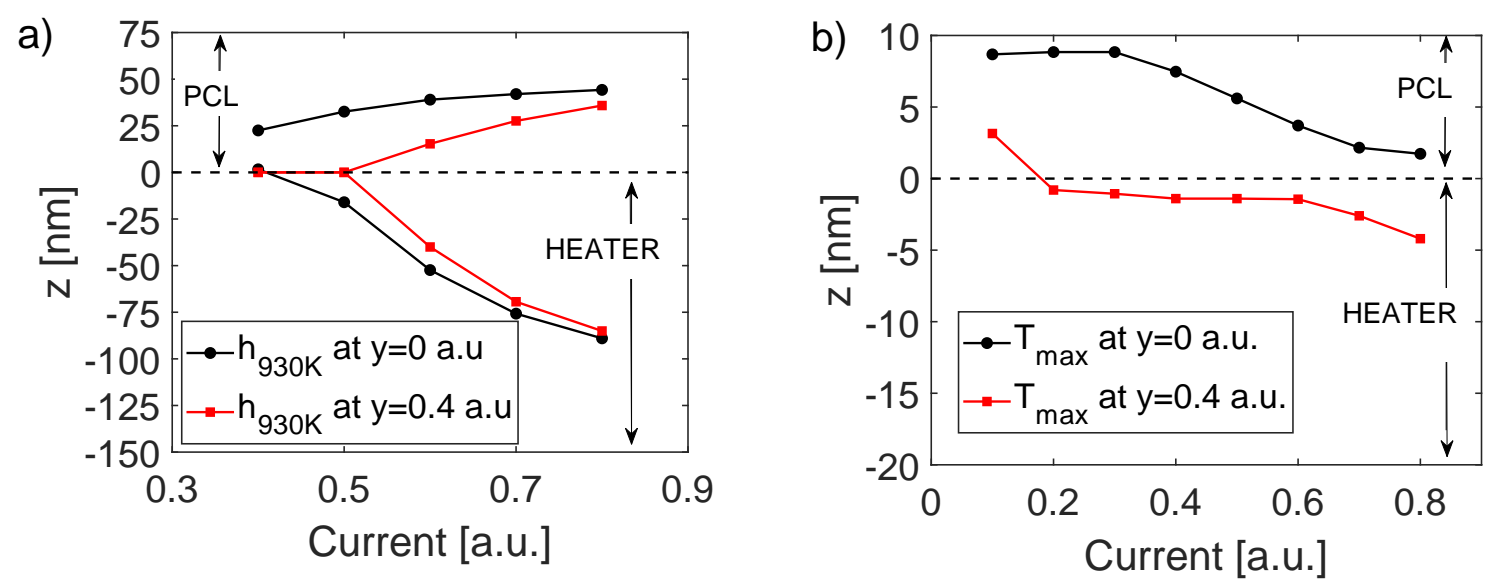

Fig. 5. Evolution of the melted region thickness $\mathrm{h}_{930 \mathrm{~K}}(\mathrm{a})$ and of the maximum temperature $\mathrm{T}_{\max }(\mathrm{b})$ achieved at the center of the cell ( $\mathrm{y}=0$ a.u.) and at the PCL/encapsulation interface ( $\mathrm{y}=0.4$ a.u.) as a function of the applied current in SiN devices. The dashed line represents the interface between the heater and the PCL.
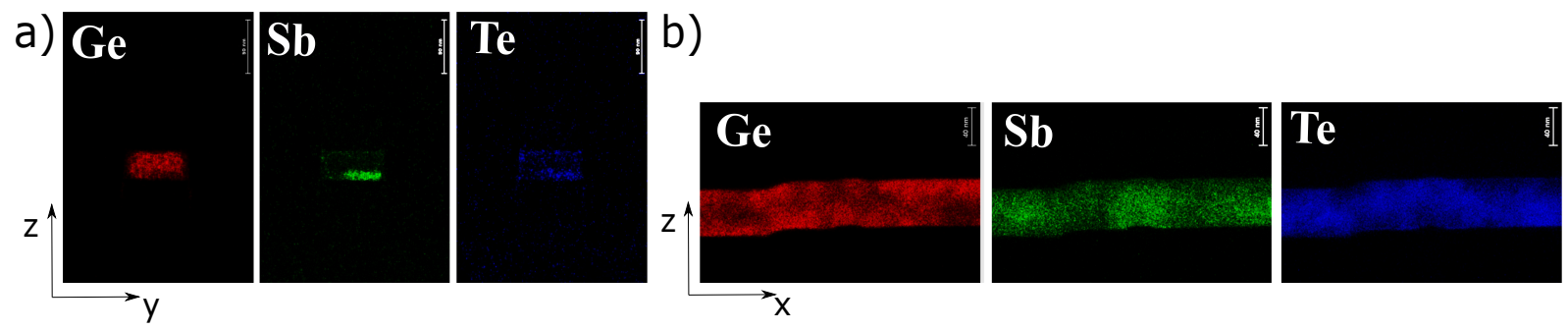

Fig. 6. TEM/EDX analyses performed on SiN encapsulated PCM programmed in RESET state. The elemental maps represent the distribution in the PCL of the three elements: Ge, Sb, Te in both zy (a) and zx (b) plane. The non uniformity of the programming in SiN devices is evidenced in particular in the zy plane analysis, where elements are not uniformly distributed over the heater/PCL interface. 

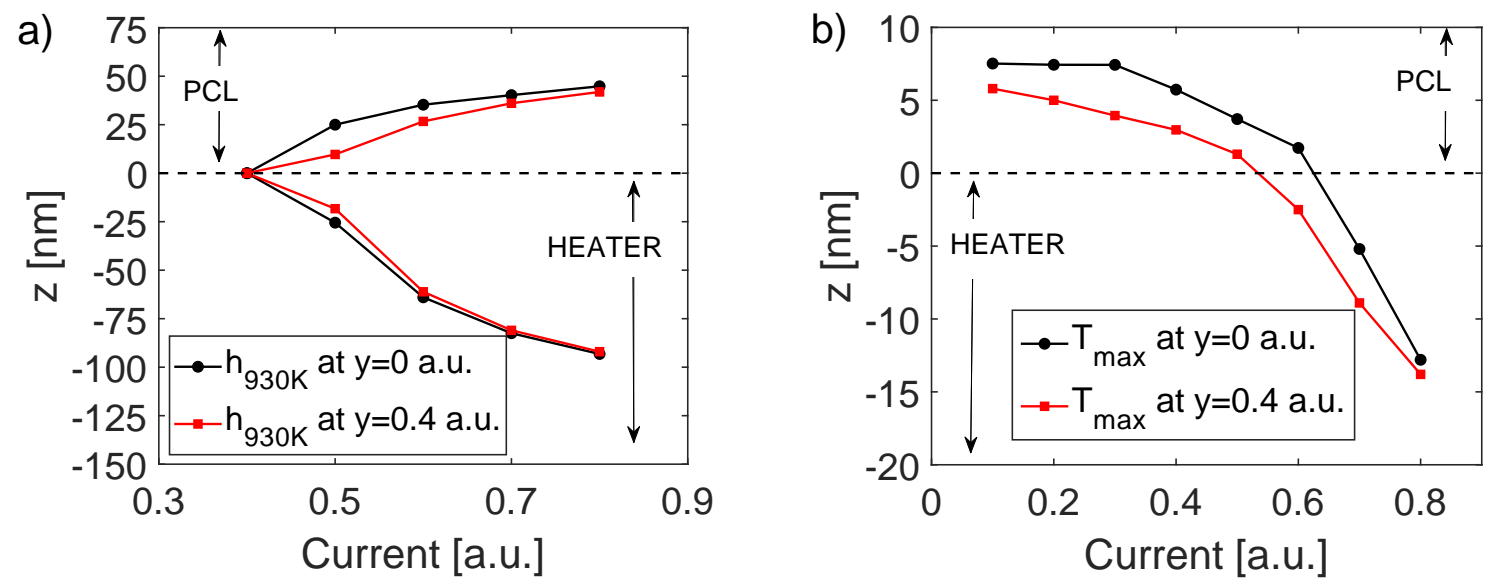

Fig. 7. Evolution of the melted region thickness $h_{930 \mathrm{~K}}$ (a) and of the maximum temperature $\mathrm{T}_{\max }(\mathrm{b})$ achieved at the center of the cell $(\mathrm{y}=0$ a.u. $)$ and at the PCL/encapsulation interface $(\mathrm{y}=0.4$ a.u. $)$ as a function of the applied current in SiC devices. The dashed line represents the interface between the heater and the PCL.
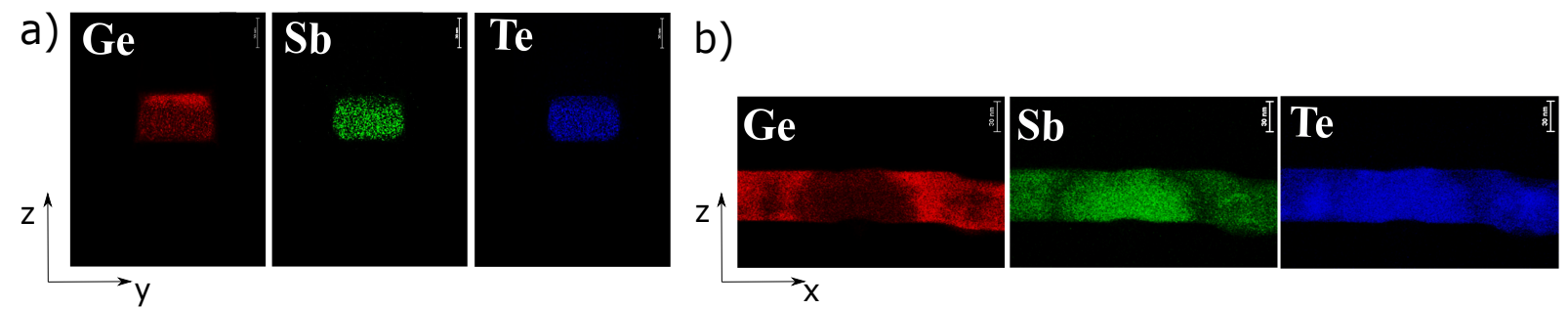

Fig. 8. TEM/EDX analyses performed on $\mathrm{SiC}$ encapsulated PCM programmed in RESET state. The elemental maps represent the distribution in the PCL of the three elements: Ge, Sb, Te in both zy (a) and zx (b) plane. A good uniformity of the temperature profile achieved in the device during the programming operation is confirmed in both planes analyses. 